\title{
Christina Plank
}

\section{Kapitalistische und territorial-geopolitische Logiken der Macht}

Die Rolle der EU im Ukraine-Konflikt ${ }^{1}$

\section{Einleitung}

Mit dem Ukraine-Konflikt hat sich seit dem Frühjahr 2014 ein Konfliktherd an den östlichen Außengrenzen der Europäischen Union (EU), also in ihrer unmittelbaren Umgebung, entwickelt. Der Konflikt forderte laut Vereinten Nationen seit April 2014 bereits an die 8.000 Menschenleben in der Ostukraine. Darüber hinaus wurden 17.800 Verletzte und über zwei Millionen Flüchtlinge, davon 1,4 Millionen Binnenflüchtlinge verzeichnet (Euronews 2015; UN News Centre 2015; Stern 2015). Auslöser der Auseinandersetzungen stellte die NichtUnterzeichnung des Assoziierungs- und des darin integrierten Freihandelsabkommen mit der EU durch den ehemaligen ukrainischen Präsidenten Wiktor Janukowytsch Ende November 2013 dar. Mit diesem Abkommen stellte die EU die Ukraine vor die Wahl, sich zwischen einer westlichen oder östlichen politischen und wirtschaftlichen Ausrichtung zu entscheiden. Sie verhinderte somit die Fortsetzung des ukrainischen außenpolitischen Lavierens zwischen Ost und West sowie deren mögliche Neutralität. Die EU trug damit wesentlich zur Eskalation des Konflikts bei. Seitdem sind die EU und ihre Mitgliedsländer bemüht, wieder Stabilität in der Region herzustellen - sei es in der Vermittlerrolle zwischen Regierung und Opposition kurz vor dem Sturz des Präsidenten Wiktor Janukowytschs im Februar 2014 oder rund um die Waffenstillstandsgespräche des Minsker Abkommens. Sollte es jedoch zu einer wie momentan geplanten Umsetzung des Assoziierungs- und Freihandelsabkommen kommen, wären diese Bemühungen des Ausgleichs vergeblich.

Die Ukraine ist als zweitgrößtes Land der ehemaligen Sowjetunion - nach Russland - nicht nur von wirtschaftlichem Interesse für den Westen und den Osten, sondern aufgrund ihrer Lage zwischen der Europäischen Union und Russ-

1 Ich danke Markus Wissen und Joachim Becker für die hilfreichen Kommentare und Anregungen. 
land vor allem auch von geostrategischer Bedeutung. Dies ist in den aktuellen Kämpfen in der Ostukraine klar ersichtlich. Vor dem Hintergrund dieser Entwicklungen gehe ich im folgenden Beitrag der Frage nach, welche Rolle die EU im Ukraine-Konflikt spielt. Meine These ist, dass im Zuge dieses Konflikts eine „territorial-geopolitische Logik“ über eine „kapitalistische Logik“ (Harvey 2003) die Oberhand gewonnen hat. Die Interessen der Kapitalfraktionen der EU-Länder an der Ukraine sind zwar vorhanden; sie reichen jedoch nicht aus, um den Konflikt in der Ostukraine vollständig erklären zu können. Vielmehr lässt sich dieser als nicht-intendierte Folge der EU-Politiken verstehen, die zu dem Konflikt mit Russland führten. Um diese Entwicklungen zu beleuchten, skizziere ich zunächst die Interessen der EU bzw. ihrer Kapitalfraktionen an der Ukraine, die sich bislang immer wieder an den Interessen der ukrainischen Oligarchie brachen. Genauer gehe ich auf die Sektoren Banken, Energie und Landwirtschaft ein, in denen sich der europäische Einfluss am stärksten manifestiert. Sodann führe ich angelehnt an David Harvey die Begriffe der kapitalistischen und territorial-geopolitischen Logik ein. Schließlich untersuche ich die Durchsetzung der territorial-geopolitischen Logik im Ukraine-Konflikt, die wesentlich durch die versuchte Integration der Ukraine in die westliche bzw. östliche Einflusssphäre bestimmt wird.

\section{Die Interessen der EU gegenüber der Ukraine}

Die Interessen der Kapitalfraktionen aus der Europäischen Union gegenüber der Ukraine scheinen auf den ersten Blick nicht evident. Denn üblicherweise werden die EU-Ukraine-Beziehungen aus dem Blickwinkel der Europäischen Nachbarschaftspolitik (ENP) und der darin verankerten Östlichen Partnerschaft, die die Beziehungen zu den östlichen Anrainerstaaten der EU regelt, beleuchtet. Die EU verfolgt darin ihr offizielles Ziel, in den Ländern der ENP „Wohlstand, Stabilität und Sicherheit aller Beteiligten zu stärken " (Europäische Kommission 2009). Die ENP hat vor allem die Implementierung bestimmter „policies“ zum Ziel, also die Übertragung des gemeinsamen Besitzstandes, des sogenannten „acquis communautaire“. Diese Übernahme wird als Demokratisierungsbestrebung der EU gerahmt (Kubicek 2005). Im Zuge dessen wird der Ukraine aber, anders als das bei den ostmitteleuropäischen Ländern durch die Osterweiterung der Fall war, kein Mitsprachrecht innerhalb der Europäischen Union gewährt. Kommissionspräsident Romano Prodi hat dies 2002 einst treffsicher mit seiner Aussage auf den Punkt gebracht, es handele sich hierbei um eine „Teilhabe an der Union mit Ausnahme ihrer Institutionen“ (Prodi zit. nach Brand 2008: 1001).

Seit der Unterzeichnung des Partnerschafts- und Kooperationsabkommens 1994 wurde der Ukraine demnach vonseiten der EU nie eine Beitrittsperspektive 
eröffnet (Wolczuk 2009: 192). Die einzelnen Mitgliedsstaaten hatten weder Interesse, für die ökonomischen Kosten eines Beitritts der Ukraine aufzukommen, noch Ambitionen, in den geopolitischen Einflussbereich Russlands einzuwirken (Bojcun 2001). So lässt sich vermuten, dass selbst nach der Westöffnung des Landes („Orangene Revolution“) „der Misserfolg Juschtschenkos in manchen Hauptstädten der EU mit Erleichterung begrüßt wurde“ (Szeptycki 2014: 196). So musste man der Ukraine weiterhin keine Mitgliedschaft in Aussicht stellen. Nichtsdestotrotz trieb die EU mittels ihrer Nachbarschaftspolitiken ihre versuchte Einflussnahme auf den ukrainischen Staat voran. Bereits in den 1990er Jahren veränderte sich die Handelsstruktur der Ukraine und öffnete sich in Richtung Westen. Der Anteil des Handels mit Russland am gesamten ukrainischen AuBenhandel sank von 80 auf 55 Prozent, mit den mittelosteuropäischen Beitrittsländern von 20 auf 10 Prozent. Der Anteil des Handels mit den EU-Ländern erhöhte sich hingegen von sechs auf 20 Prozent. Handelsdefizite erwirtschaftete die Ukraine allerdings gleichermaßen gegenüber Russland wie gegenüber der EU. Gleichzeitig herrschten in den Bereichen Chemikalien, Nahrungsmittel, Textilien, in denen die ukrainische Industrie wettbewerbsfähig gewesen wäre, Importbeschränkungen vonseiten der EU vor, die eine Modernisierung dieser Industrien verhinderten (Bojcun 2001: 6ff.).

Ab Mitte der 1990er Jahre richtete die Ukraine unter Leonid Kutschmas „multivektoraler" Politik, die zwischen West und Ost lavierte, zunächst ihren außenpolitischen Kurs zumindest deklaratorisch auf den Westen aus, um ein Gegengewicht zur historisch bedingt starken Anbindung an Russland zu erwirken. Sie artikulierte ihren Beitrittswunsch in den von Kutschma erlassenen präsidentiellen Dekreten, konkret in der Strategie der Integration der Ukraine in die Europäische Union 1998 und dem Programm der Integration der Ukraine in die EU 2000 (MFA 2012).

Durch die Herausbildung und Festsetzung der oligarchischen Strukturen seit der Unabhängigkeit des Landes erwies sich die Ukraine jedoch lange als eher unattraktiv für die EU. Diese Strukturen sind bei der Eroberung neuer Absatzmärkte und Produktionsstätten in der Ukraine besonders hinderlich und verursachen unter anderem, dass die Forderungen der EU nicht mit gewünschter Geschwindigkeit und Intensität implementiert werden, falls sie gegen die Interessen der Oligarchen verstoßen. Denn letztere können aufgrund ihrer ökonomischen und politischen Beziehungen entweder selbst in der Funktion als Abgeordnete oder über Parteien mehr oder weniger direkt auf den Staatsapparat zugreifen und durch die Kontrolle der Medien die Gesellschaft beeinflussen (Becker 2015: 409f.). Trotz deklaratorischer außenpolitischer Ausrichtung auf die Europäische Union ermöglichte die Politik des Präsidenten Leonid Kutschmas ab Mitte der 1990er Jahren den Aufbau der Oligarchie als nationale Bourgeoisie und war 
damit darauf ausgerichtet, ausländischen Akteuren eine nicht zu bedeutende Rolle einzuräumen (Müller 2015: 138; Plank 2014a: 45f.). Somit war im Gegensatz zu den Wirtschaftsmodellen der mittelosteuropäischen Staaten, wo sich westeuropäisches Kapital entscheidend einkaufte - Jan Drahokoupil spricht dort von der Entstehung eines „Kompradoren-Servicesektor“ (zit. nach Becker 2008: 6) - ausländisches Kapital unter Kutschma nur schwach in der Ukraine vertreten. Dies änderte sich mit der Orangenen Revolution und der dadurch losgetretenen Öffnung hin gegen Westen.

Obgleich eine grobe Zuordnung der Phasen unter den Präsidenten Wiktor Juschtschenko (Öffnung zum Westen) und Wiktor Janukowytsch (Ausrichtung auf den Osten, allerdings mit dem Ziel einer „Schaukelpolitik“ zwischen EU und Russland) möglich ist, lassen sich die einzelnen Oligarchen nicht eindeutig „westlichen“ oder „östlichen“ Interessen zuordnen. Sie wechseln die Seite, unterstützen auch mehrere Fraktionen gleichzeitig und haben insgesamt Machtgewinn, -ausweitung bzw. -erhalt zum Ziel. Anstelle einer eindeutigen Teilung des Landes in Kapitalfraktionen bzw. einzelnen Wirtschaftsbranchen, die sich entlang der West- und Ostukraine spalten oder zur Europäischen Union oder Russland zuordnen lassen, sind die ukrainischen Oligarchen nach regionalen Clans gegliedert und als unterschiedliche Finanz-Industrie-Gruppen zu verstehen. Hier haben sich Dnipropetrowsk und Donezk als die heute weiterhin wichtigsten Zentren behauptet. Die Oligarchen entstanden in erster Linie durch die Aneignung staatlicher Betriebe in den Bereichen Schwerindustrie, Energiewirtschaft und Banken, diversifizieren und investieren aber auch in andere Branchen wie beispielsweise in die immer bedeutsamer werdende Landwirtschaft (Yurchenko 2012; Plank/ Plank 2014; Matuszak 2012: 14f.).

Mit dem 2014 ratifizierten Assoziierungs- und Freihandelsabkommen wird weiterhin die Übernahme des gemeinsamen Besitzstandes - dieses Mal sollen es bis zu 80 Prozent sein - in die Ukraine exportiert (Böttger 2014: 96). Dadurch erfolgt nicht nur eine Liberalisierung des Handels, sondern auch eine Übertragung von Standards, die EU-Unternehmen den Zutritt zum ukrainischen Markt ermöglichen sollen. Ein Blick auf die Außenhandelsstatistik zeigt allerdings, dass die Ukraine bislang sowohl mit dem westlichen als auch mit östlichen Nachbarn wirtschaftlich stark verwoben ist. 2012 stammten 40,7 Prozent der gesamten ukrainischen Importe aus der EU und 19,6 Prozent aus Russland, wobei hier speziell die Energieimporte aus Russland von großer Bedeutung sind. Die ukrainischen Exporte gingen jeweils zu einem Viertel in die EU und nach Russland. Dabei liefert die Ukraine vor allem Rohstoffe in die EU, während sie nach Russland weiterverarbeitete Produkte exportiert. Eine einseitige Ausrichtung der ukrainischen Wirtschaft auf die EU würde ihr demnach schaden und nicht zu einer positiven Entwicklung beitragen (EuroMemorandum 2015: 44). Indem die EU 
ein Assoziierungs- und Freihandelsabkommen mit der ökonomisch schwächeren Ukraine schließt, verspricht sie, ähnlich wie bei Abkommen des Internationalen Währungsfonds (IWF), dass nach einer möglichen schwierigen Übergangsphase positive Effekte in der Zukunft eintreten, die zu einer Modernisierung des Landes führen würden. Aufgrund der ungleichen Austauschverhältnisse (verarbeitete Produkte vs. Rohstoffe) sind jedoch eher eine „Vertiefung der DeIndustrialisierung und vertiefte Abhängigkeitsstrukturen" (Becker 2014: 2) zu erwarten, die einer Entwicklung des Landes im Wege stehen.

Wie genau bzw. in welchem Ausmaß die momentan herrschenden ukrainischen Gruppen sich mit den EU-Interessen bzw. jenen des Westens verbinden, um daraus Profit zu schlagen, ist derzeit schwer abzuschätzen. Die Schwerindustrie im Osten musste bereits durch den Konflikt, der in jener Region ausgetragen wird, in dem sich die Produktionsstätten befinden, schwere Einbußen hinnehmen. Darüber hinaus wird erwartet, dass sie durch das Assoziierungs- und Freihandelsabkommen mit weiteren Verlusten zu rechnen hat, wohingegen die Agrarindustrie davon profitieren dürfte (Becker 2015: 421). Feststellen lässt sich jedoch, dass die Westausrichtung des Landes, wie sie bereits unter Juschtschenko nach der Orangenen Revolution eingeleitet wurde, von der neuen ukrainischen Regierung mit großem Nachdruck fortsetzt wird.

\section{EU-Interessen an einzelnen Sektoren der ukrainischen Wirtschaft}

In diesem Abschnitt betrachte ich drei Branchen (Banken, Energie und Landwirtschaft), die sich für die EU bzw. deren Kapitalfraktionen in der Ukraine als zentral herauskristallisieren. Die EU bemüht sich hier um die Eroberung neuer Märkte und den Zugriff auf Ressourcen. Seit der Orangenen Revolution sind zahlreiche Banken verschiedener EU-Länder prominent in der Ukraine vertreten. So haben in weniger als fünf Jahren ausländisch geführte Banken über 50 Prozent des Sektors vereinnahmt. Der Anteil der vom ukrainischen Staat geführten Institute am gesamten Banken-Sektor des Landes schrumpfte auf weniger als ein Achtel, so die Europäische Bank für Wiederaufbau und Entwicklung (nach Mykhnenko/Swain 2010: 158). An dieser Verteilung änderte auch die Wirtschafts- und Finanzkrise im Grundsatz nichts. 2011 betrug der Anteil der ausländischen Anleger noch immer 37 Prozent, wobei neben den westlichen Banken hier auch russische eine wichtige Rolle spielten. Der Bankenbereich zeichnet sich zudem durch eine „relativ geringe Präsenz der Oligarchen“ (Matuszak 2012: 46) aus. Nur die PrivatBank mit Ihor Kolomojskyj und Hennadij Boholjubow (Dnipropetrowsk Clan) auf Platz eins und Rinat Achmetows FUIB (First Ukrainian National Bank) (Donezk Clan) auf 
Platz neun wurden 2011 von ukrainischen Oligarchen kontrolliert. Die Raiffeisen Bank Aval der österreichischen Raiffeisenbank International AG, die UkrSibbank der französischen BNP Paribas Group, die Ukrsocbank der italienischen Bank Austria und UniCredit Group, die ungarische OTP Bank, das Bank Forum der deutschen Commerzbank und die ING Bank der holländischen ING Bank H.B. zählten zu den zwanzig größten Banken der Ukraine. Zwar haben einige Banken wie beispielsweise die schwedische Swedbank, die Skandinaviska Enskilda Banken und die Commerzbank, in Folge der Wirtschafts- und Finanzkrise seit 2009 Anteile verkauft oder beabsichtigen, aufgrund der Verluste bzw. der unsicheren Investitionslage ihr Engagement zu reduzieren. Dabei stehen sie allerdings oft vor der Schwierigkeit, geeignete Käufer zu finden (Matuszak 2012: 46ff.; SpiegelOnline 2012). Insgesamt kann man in den letzten Jahren eine Verschiebung zugunsten der russischen Banken wie der staatlichen Prominvestbank und der Sberbank feststellen, die auch schon vor der Krise vertreten waren (Nationalbank der Ukraine 2015). Kredite des IWF sollen darüber hinaus Banken der Oligarchen Ihor Kolomojskyj, Rinat Achmetow, Wiktor Pintschuk (Dnipropetrowsk Clan) stützen, um dazu beizutragen, das ukrainische Bankensystem zu konsolidieren (Helmer 2015). In diesem Zusammenhang ist zu betonen, dass das Hilfsprogramm der Europäischen Union im Umfang von elf Milliarden Euro neben den 140 Millionen Euro humanitäre Hilfe als Mikro-Finanzhilfe an die Erfüllung der IWF-Forderungen gekoppelt ist (EEAS o.J.).

Eine weitere Branche, die einen Schwerpunkt der IWF-Programme, des Reformprogramms der ukrainischen Regierung sowie für die EU darstellt, ist die Energiewirtschaft. Lange flossen 80 Prozent des von Russland an die EU gelieferten Erdgases über Pipelines quer durch die Ukraine, wodurch diese als Gas-Transitland von enormer Bedeutung war. Seit geraumer Zeit versucht die EU jedoch, mittels materieller und räumlicher Diversifizierung ihre Abhängigkeit von importierten Energieträgern zu reduzieren, und Russland bemüht sich, alternative Transitrouten zu erschließen, die nicht durch die Ukraine führen. Deutschland ist seit 2011 über die Nord Stream Pipeline direkt mit Russland verbunden und umgeht somit die Ukraine (Schubert et al. 2014: 2). Außerdem wird mittels neuer Technik Erdgas in die Ukraine über die EU geliefert - 2014 waren es etwa ein Viertel der Importe (Europäische Kommission 2015). Nichtsdestotrotz ist und bleibt die Kontrolle des Sektors bzw. der Infrastruktur zentrales Thema für die Europäische Union. Daher schloss die EU bereits 2005 mit der Ukraine im Bereich Energie eine gemeinsame Absichtserklärung. Unter Präsident Wiktor Jaunkowytsch wurde das Land 2011 Mitglied der Europäischen Energiegemeinschaft. Diese wurde 2005 ursprünglich im Hinblick auf die südosteuropäischen Staaten gegründet und dient dazu, die Übernahme des acquis im Bereich Energie unter ihren Mitgliedern zu forcieren und die Liberalisierung des Energiesektors 
voranzutreiben (Szeptycki 2014: 191ff.). Auch das Assoziierungsabkommen beinhaltet ein eigenes Kapital zu Energie mit dem Ziel, eine unabhängige Regulierungsbehörde für Gas und Elektrizität einzurichten sowie die Senkung von Energiesubventionen für Haushalte durchzusetzen (EuroMemorandum 2015). Diese Ziele sollen dazu beitragen, den Energiesektor oligarchischer Monopolkontrolle zu entreißen und ihn international wettbewerbsfähig zu machen. Mit Ukrnafta, dem größten staatlichen Ölunternehmen der Ukraine, das sich bis im Frühjahr 2015 noch faktisch in der Hand Kolomojskyjs befand, wurde bereits ein Anfang gemacht. Ebenso wurden Gesetze in Übereinstimmung mit dem Dritten Energiepaket der EU (von 2009) verabschiedet, die die Kontrolle von Oligarch Dmitry Firtasch, der im Nahverhältnis zu Gazprom steht, über Naftogaz einschränken sollen. Als Nächstes wird angestrebt, Achmetows Monopol DTEK am Elektrizitätsmarkt aufzubrechen (Kyiv Post 2015: 26ff.). Zudem hat die ukrainische Regierung insgesamt die Privatisierung von 350 Staatsunternehmen ins Auge gefasst. Energieunternehmen und Kraftwerke aber auch andere Objekte wie der Hafen in Odessa sollen (unter Ausschluss von russischem Kapital) an Unternehmen aus den USA und der EU gehen, um die leeren Staatskassen zu füllen (Die Presse 2015a; Die Presse 2015b).

Als dritter Sektor, der auf wachsendes Interesse bei der EU stößt, ist die Landwirtschaft zu nennen. Sie verspricht hohe Gewinne. Die ukrainische Stahl- und Kohleproduktion hingegen ging um 17 bzw. 22 Prozent im Vergleich zum letzten Jahr zurück (Rachkevych 2015: 14). Die Landwirtschaft stellt seit Beginn der 2000er Jahre, aber insbesondere seit der Wirtschafts- und Finanzkrise, ein immer profitableres Geschäftsfeld dar und wird darüber hinaus im Assoziierungs- und Freihandelsabkommen anvisiert. Dieses unterstützt vor allem den Export von Agrarrohstoffen und Nahrungsmitteln in die EU (EU 2015). Dadurch fördert es die Entwicklung der Ukraine als Rohstoffproduzentin und -exporteurin, wovon wiederum jene Oligarchen profitieren, die in der Agrarindustrie tätig sind (EuroMemorandum 2015: 44). Dazu zählt der „Schokoladenkönig“ und derzeitige Präsident Petro Poroschenko aber auch Achmetow und Kolomojskyj mischen hier mit ihren über 100.000 Hektar großen Agroholdings HarvEast und der PrivatAgroholding mit (Latifundist 2015). Viele der Agroholdings in der Ukraine, bei denen es sich um vertikal und horizontal integrierte Agrarbetriebe handelt, die oftmals aus ehemaligen Kolchosen bestehen, werden mittels Krediten der Europäischen Bank für Wiederaufbau und Entwicklung (EBWE) unterstützt und sind an europäischen Börsen notiert (Plank/Plank 2014). Diese fördern damit einhergehend die Industrialisierung der Landwirtschaft und tragen dazu bei, dass die Agroholdings die landwirtschaftlichen Flächen in der Ukraine kontrollieren. Außerdem liefern sie Expertise im Bereich Privatisierung von Land und assistieren damit bei der Öffnung des ukrainischen Bodenmarkts (Plank 2015). 
Auch im landwirtschaftlichen Sektor werden von der ukrainischen Regierung Privatisierungen von Staatsunternehmen unter europäischer Anleitung anvisiert oder um es mit den Worten des aktuellen ukrainischen Landwirtschaftsministers auszudrücken: „Wir werden uns nicht auf unseren Lorbeeren ausruhen - wir werden weiter die Industrie deregulieren und die Privatisierung europäischer Gesellschaften sowie die gesetzgeberische Arbeit in Richtung europäische Integration fortsetzen." (Pavlenko 2015)

Zusammenfassend ist daher festzustellen, dass die ökonomischen EU-Interessen seit der Westöffnung Juschtschenkos versucht haben, im Land Fuß zu fassen, dies aber bisher nur in eingeschränktem Maße gelang - mit Ausnahme der Bankenbranche. Der Energiesektor befand sich bislang in der Hand der ukrainischen Oligarchie. Die Politiken der EU in Kombination mit den Forderungen des IWF scheinen hier jedoch eine Umstrukturierung des Sektors zu initiieren, die gleichzeitig von den herrschenden Gruppen in der Ukraine genutzt werden könnten, um sich gegenüber ihren konkurrierenden oligarchischen Kollegen durchzusetzen. Die Landwirtschaft weckt schließlich mittels Kontrolle des Bodens sowie der gesamten agrarischen Wertschöpfungskette neue Profithoffnungen bei Oligarchen wie auch bei der EU. Wenngleich der wieder eingeschlagene Kurs den Interessen des europäischen Kapitals zugutekommen dürfte, können die europäischen ökonomischen Interessen für sich alleine betrachtet den UkraineKonflikt nicht erklären. Stattdessen liefern die oligarchischen Strukturen eine bestimme Eigendynamik, die den Konflikt im Osten des Landes beeinflussen und daher mit zu berücksichtigen sind.

\section{Die kapitalistische und territorial-geopolitische Logik der Macht}

Die Rolle der EU im Ukraine-Konflikt lässt sich zudem nicht nur auf Kapitalinteressen zurückführen, die versuchen, auf den ukrainischen Staat zuzugreifen. Vielmehr ist die territoriale Komponente ausschlaggebend, um den Konflikt zu verstehen. In den Worten von Klaus Müller (2015: 136) geht es um das „Zusammenspiel oligarchischer Machtstrategien, identitätspolitischer Mobilisierung und geopolitischen [sic] Rahmenbedingungen“, ergänzt um die in den vorangegangenen Abschnitten betrachteten ökonomischen Strategien der EU. Im Folgenden beziehe ich mich daher auf David Harveys Verständnis des Zusammenspiels kapitalistischer und territorial-geopolitischer Logiken, um die Bedeutung der EU im Konflikt erklären zu können.

Um die krisenhaften Tendenzen des Kapitalismus zu beschreiben, unterscheidet Harvey im Anschluss an Giovanni Arrighi zwischen den beiden Logiken der 
Macht, die die beiden Teile des „kapitalistischen Imperialismus“(Harvey 2003: 26) bilden und denen unterschiedliche Interessen zugrunde liegen. Unter „territorialer Logik“ versteht Harvey „politische, diplomatische und militärische Strategien, die von einem Staat geltend gemacht und verwendet werden“ (ebd.: 26). Die „kapitalistische Logik“ umfasst wiederum die „Wirtschaftsmacht, die quer durch ein durchgehendes Raumkontinuum hin zu oder weg von territorialen Einheiten fließt" (ebd.: 26) und die sich beispielsweise im Handel, in Kapitalströmen oder in Währungsspekulationen äußert. Die Interessen des Kapitals sind von Profitmaximierung bestimmt. Politiker_innen hingegen sorgen sich in erster Linie um den Erhalt und Ausbau von Macht. Harvey zufolge scheinen letztere im weitesten Sinne für das angebliche Allgemeinwohl oder eben die Interessensbefriedigung gewisser Eliten verantwortlich zu sein, verfügen über ein Territorium und sind im Falle von demokratischen Staaten an Wahlen gebunden. Im Gegensatz dazu unterliegen Unternehmen keinem demokratischen Machtwechsel und Kapital kann - im Unterschied zu den an Territorien gebundenen Staaten - Räume erobern bzw. ebenso rasch wieder aus diesen abgezogen werden (ebd.: 27).

Zugleich spielen Staaten oder auch eine Gruppe von Staaten wie die Europäische Union eine wichtige Rolle in der Absicherung des Kapitalismus mittels „institutioneller Strukturen des Rechts, des Privateigentums, der Verträge und der Sicherheit der Geldform" (Harvey 2003: 89). Harvey bezeichnet in diesem Zusammenhang den bürgerlichen Staat als präferierte Form, um Klassenkonflikte zu bearbeiten: „Kapitalisten benötigen einen solchen Rahmen nicht unbedingt, um zu funktionieren, aber ohne ihn sehen sie sich größeren Risiken ausgesetzt." (Ebd.: 90) Die Aufrechterhaltung des kapitalistischen Systems wird daher maßgeblich durch den Staat unterstützt.

Wenn Kapital sich innerhalb eines Staates nicht mehr profitabel verwerten lässt, sucht es nach anderen Räumen, die profitabel erscheinen. Die Einflussnahme bzw. die Kapitalverwertung in den neu erschlossenen Räumen erfolgt oftmals über die kapitalistische Logik, also etwa über Handelsverträge oder Investitionsabkommen. Diese spiegelt sich im Assoziierungs- und Freihandelsabkommen der Europäischen Union mit der Ukraine wider. Die Risiken, in der Ukraine tätig zu werden, waren für die Kapitalfraktionen der EU bislang hoch, da die herrschende Klasse dort mit der Staatsklasse eins zu eins verbunden und damit auch keine relative Autonomie des Staates im Sinne Poulantzas' vorhanden war (van der Pijl 2015: 57; Becker 2015: 410). Dies verunmöglichte bzw. erschwerte den Zugriff außenstehender Kräfte auf den ukrainischen Staat, der mittels eines neuen Assoziierungsabkommens erleichtert werden soll. Die Herstellung von Konsens ist hierbei wichtiges Mittel, um, wie Harvey es für die Zeit des Kalten Krieges beschrieb, die „privaten Haus- und Grundstückbesitzenden“ (Harvey 2003: 39) gegen den Sozialismus zu vereinigen, in diesem Falle gegen 
das post-sozialistische und korrupt-oligarchische System sowie die ineffizienten Staatsbetriebe. Eine andere Möglichkeit der Durchsetzung von Interessen ist die Geopolitik, wobei „die geopolitischen Konflikte fast notwendigerweise aus den molekularen Prozessen der Kapitalakkumulation entstehen“ (ebd.: 107). Dementsprechend setzte Russland auf einen geopolitischen Kampf, um seine Einflusssphäre in der Ukraine zu verteidigen.

Die kapitalistische und territorial-geopolitische Logiken der Macht sind daher nicht als getrennt voneinander zu verstehen, sondern sie sind miteinander verwoben. Harvey bezeichnet die Beziehung der Logiken der Macht auch als "eher oft widersprüchlich (sprich dialektisch) als funktional und einseitig" (ebd.: 30). Dies führt nicht zuletzt dazu, dass diese auch gänzlich im Widerspruch zueinanderstehen können (ebd.: 29). In Bezug auf den Ukraine-Konflikt äußert sich dieser Widerspruch, der zwischen den beiden Logiken herrscht, offen im Handeln der Europäischen Union gegenüber Russland. Die wirtschaftlichen Beziehungen der EU zu Russland, die sie über jene stellt, die sie mit der Ukraine führt, sind durch die militärischen Auseinandersetzungen in der Ostukraine gefährdet. Die ökonomischen Interessen der EU stehen im Ukraine-Konflikt daher den territorial-geopolitischen diametral gegenüber.

\section{Der Ukraine-Konflikt als nicht-intendierte Folge der EU-Nachbarschaftspolitiken}

Die Nicht-Unterzeichnung des Assoziierungs- und Freihandelsabkommens durch Wiktor Janukowytsch im November 2013 löste eine Kette von Ereignissen aus, mit denen die Europäische Union in keiner Weise gerechnet hatte. Diese verliefen über die Proteste am Majdan, die bis im Februar 2014 andauerten, die Abspaltung der Krim im März 2014, bis zum seit April 2014 andauernden Konflikt in der Ostukraine, der sich in die lange Reihe eingefrorener Konflikte im postsozialistischen Raum eingereiht hat (Bergkarabach, Transnistrien, Abchasien und Südossetien). Der in den beiden Minsker Abkommen vom September 2014 und Februar 2015 vereinbarte Waffenstillstand zwischen den „pro-ukrainischen“ und „pro-russischen“ Parteien wurde immer wieder gebrochen. Der ukrainische Staat hat im Osten des Landes sein Gewaltmonopol verloren und die territoriale Integrität der Ukraine, die durch das derzeit oft in den Medien zitierte Budapester Memorandum von 1994 gewährleistet werden sollte, ist seit dem Konflikt nicht mehr gegeben. Das Abkommen wurde damals von der Ukraine, den USA, Großbritannien und Russland unterzeichnet - als Gegenleistung dafür, dass die Ukraine die bis in die 1990er Jahre in ihrem Besitz befindlichen Atomwaffen an Russland auslieferte (Council on Foreign Relations 1994). 
Die Politik der Europäischen Union gegenüber der Ukraine bzw. gegenüber Russland wird etwa als naiv eingeschätzt (vgl. Jopp/Böttger 2014) oder mit der zugespitzten Aussage kommentiert, dass der Westen am Konflikt schuld sei (vgl. Mearsheimer 2014). Dagegen vertrete ich die These, dass die Verschärfung der Situation als nicht-intendierte Folge der Nachbarschaftspolitiken der EU zu verstehen ist. Darin hat die territorial-geopolitische gegenüber der kapitalistischen Logik an Bedeutung gewonnen und sich gegenüber dieser durchgesetzt. Die EU hat die Reaktion der Ukraine als auch Russlands zur Verteidigung seiner Interessen im „nahen Ausland“ einerseits unterschätzt, andererseits hat sich ihre Politik verselbstständigt. So bot die Europäische Union der Ukraine das Assoziierungs- und Freihandelsabkommen nach den Majdan-Protesten erneut zur Unterschrift an, woraufhin der politische Teil des Abkommens im März und der wirtschaftliche Teil im Juni 2014 von der Regierung Arsenij Jazenjuk und Petro Poroschenkos unterzeichnet wurden. Im September 2014 ratifizierten das ukrainische und das Europäische Parlament das Abkommen. Im Januar 2016 soll es trotz Russlands Widerspruch in Kraft treten. Obgleich die wirtschaftlichen Fragen im Zentrum des Assoziierungs- und Freihandelsabkommens stehen, ist im politischen Teil von militärischer Zusammenarbeit in Sicherheitsfragen die Rede, was die geopolitische Komponente des Dokuments unterstreicht und dazu beiträgt, den Konflikt zu verstetigen (EuroMemorandum 2015: 46).

Die Annäherung der Ukraine an die NATO, und damit der Schritt in Richtung Integration in die euroatlantische Einflusssphäre, stellte bereits in den 1990er Jahren ein Vordringen ins russische „nahe Ausland“ dar. 1994 trat die Ukraine als erster GUS-Staat dem „Partnership for Peace Programm“ bei. 1997 wurden die Beziehungen durch eine „besondere Partnerschaft“ vertieft (NATO 2015), die 2003 bis hin zur Entsendung ukrainischer Soldaten in den Irak führte (Spinner 2005). Trotzdem herrschte lange ein historisch bedingtes Naheverhältnis zwischen der Ukraine und Russland im Bereich Militär. Die Ukraine äußerte zunächst nicht den Wunsch, NATO-Mitglied zu werden (Bojcun 2001: 8). Ab 2004 strebte Juschtschenko allerdings nicht nur eine Integration in die EU an, sondern auch in die NATO, wohingegen Janukowytsch sich 2010 als erster ukrainischer Präsident offiziell gegen den NATO-Kurs stellte und die Stationierung der russischen Schwarzmeerflotte auf der Krim für weitere 25 Jahre im Gegenzug für billigeres russisches Gas verlängerte (EurActiv 2010). Die NATO genoss lange Zeit in der ukrainischen Bevölkerung kein hohes Ansehen, auch aufgrund des Einsatzes in Serbien Ende der 1990er Jahre. Dies hat sich allerdings im Zuge des jüngsten Konflikts verändert. Während sich im April 2012 noch nicht einmal 15 Prozent der ukrainischen Bevölkerung für einen NATO-Beitritt ausgesprochen haben, waren es im Juli 2015 bereits 64 Prozent (Zolinka 2015). 
Insgesamt war das Interesse der USA, die Ukraine - sowie generell den gesamten früher unter Einfluss der Sowjetunion stehenden Raum - in ihren geostrategischen Einflussbereich zu bringen, größer als das Interesse der EU, die Ukraine ökonomisch zu integrieren. Dies äußerte sich auch darin, dass die Ukraine in den 1990ern der drittgrößte Empfänger von US-Entwicklungshilfegeldern war (Bojcun 2001: 8). Außerdem weitete die NATO ihren Einflussbereich mit dem Beitritt von Polen, Tschechien und Ungarn 1999 und der baltischen Staaten, Bulgarien, Rumänien, Slowakei und Slowenien 2004 wesentlich aus.

Noch bis vor Kurzem schien es so, als ob die EU die Einflusssphäre Russlands im GUS-Raum respektierte. Sie wollte augenscheinlich nicht riskieren, ihre „Russland zuerst“-Politik, die Russland vor alle anderen Ost-Staaten stellte, zu unterwandern. Denn „[die] Ukraine, Belarus, die Republik Moldau sowie die anderen postsowjetischen Republiken im Südkaukasus und in Mittelasien gelten allgemein als viel zu unwichtig, um deswegen eine 'Verstimmung Russlands' oder sogar - wie nicht wenige Beobachter zu glauben scheinen - die Versorgung mit russischen Energieträgern zu riskieren." (Malek 2010: 179) Ein Grund, der zur Veränderung der Kräfteverhältnisse innerhalb der EU, und damit zu deren Positionierung gegenüber Russland bzw. den USA beitrug, war der Beitritt der ostmitteleuropäischen Staaten zur EU. Mit der Osterweitung 2004 erhielt die EU nicht nur eine gemeinsame Außengrenze mit der Ukraine, auch das Verhältnis zu Russland veränderte sich durch die neuen Mitgliedsländer. Polen und die baltischen Länder wie später auch Rumänien sind Anhänger einer unilateralen von den USA dominierten Weltordnung. Dies hängt eng mit ihrer historischen Erfahrung mit der Sowjetunion zusammen. Dabei bleiben jedoch auch ihre Abhängigkeiten von Energieressourcen aus dem post-sowjetischen Raum groß. Damit stärken die neuen Mitgliedsländer die an den USA orientierte Fraktion, zu der Großbritannien zählt, welche Frankreich und Deutschland gegenübersteht, die für einen eigenständigeren Kurs der EU eintreten (Becker 2008).

So ist die EU-Politik gegenüber der Ukraine ein Resultat von teilweise widersprüchlichen Interessen der Nationalstaaten und Kapitalfraktionen. Diese sind auch im Umgang mit den wirtschaftlichen Sanktionen gegenüber Russland ersichtlich, die als Reaktion auf den Konflikt verhängt wurden. Die EU sprach Einreiseverbote gegenüber ukrainischen und russischen Politikern aus und fror deren Konten ein. Die Sanktionen beinhalten darüber hinaus Beschränkungen im Bereich Waren und Dienstleistungen im Energiesektor, ein Waffenembargo, beschränkten Zugang zu den Kapitalmärkten und Handels- und Investitionsbeschränkungen für die Krim und Sewastopol (GTAI 2015). Deutschland stimmte eher widerwillig zu, weil es seine wirtschaftlichen Beziehungen zu Russland, insbesondere in Energie-Fragen, nicht zu sehr aufs Spiel setzen wollte. Aber auch Österreich, Italien, Tschechien, Ungarn und die Slowakei waren zunächst 
dagegen, wohingegen Großbritannien, Polen, Rumänien und die Balkanländer für Sanktionen eintraten (EuroMemorandum 2015: 44). Die Zusammenarbeit mit Russland im Bereich Atomenergie in Ungarn, in der Rüstungsindustrie in Frankreich und mit den Banken in Österreich erklären die Ausnahmen bei den Sanktionen gegenüber Russland (Brangsch 2015). Russland antwortete auf die EU-Sanktionen mit Agrar- und Lebensmittelimportstopps aus der EU, den USA, Kanada, Norwegen und Australien sowie mit Einreiseverboten gegenüber europäischen Politikern. Überdies kündigte es an, die Sanktionen auf die Ukraine auszuweiten, sollte sie den wirtschaftlichen Teil des Assoziationsabkommens realisieren. Der europäischen Landwirtschaft bereiten die Sanktionen erhebliche Probleme (GTAI 2015).

\section{Schlussfolgerungen}

Die Erweiterungspolitiken der Europäischen Union, die in der Unterzeichnung des Assoziierungs- und Freihandelsabkommens gipfelten, hatten zum Ziel, die Ukraine vor allem in den wirtschaftlichen Einflussbereich des Westens zu bringen. Die Erschließung des ukrainischen Raums durch das Abkommen soll der Verwertung des europäischen Kapitals dienlich sein. Die russischen Interessen wurden dabei jedoch missachtet, wodurch ein geopolitisch-militärischer Konflikt hervorgerufen wurde, der in der Ostukraine ausgetragen wird.

Die kapitalistische Logik schlug für die EU in nicht-intendierter Weise im Moment der Konflikte um die Unterzeichnung des Assoziierungs- und Freihandelsabkommens in die territorial-geopolitische Logik um. Diese wurde durch die US- und NATO-Bemühungen verstärkt und richtet sich damit teilweise gegen sich selbst, weil nun durch die betriebene Sanktionspolitik der EU die europäischen Wirtschaftsinteressen gegenüber Russland wesentlich beeinträchtigt werden. Die EU hatte die Folgen rund um das Angebot und die Unterzeichnung des Assoziierungs- und Freihandelsabkommens mit der Ukraine insgesamt maßgeblich unterschätzt. „Erst spät ist europäischen Politikern aufgegangen, dass die Alternativen des Assoziierungsabkommens zu riskant formuliert waren." (Müller 2015: 150f.) Der Konflikt kann daher als nicht-intendierte Folge der EU-Nachbarschaftspolitiken gefasst werden und er kann nur beendet werden, wenn die EU ,auf das Assoziationsabkommen in der vorgesehen Form verzichten und sich auf ein politisch tiefer gehängtes Kooperationsabkommen beschränken [würde], das Kompromiss-Spielräume in der Ukraine schaffen könnte." (Becker 2014: 3) Diese Schaffung von Kompromissen wäre notwendig. Die Entschärfung des Konflikts setzt eine Stellung der Ukraine als neutraler Staat zwischen West und Ost voraus. 


\section{Literatur}

Becker, Joachim (2008): Der Drang nach Osten: Wirtschaftliche Interessen und geopolitische Strategien. In: Kurswechsel 4: 5-29.

- (2014): Die EU-Strategie im Ukrainekonflikt. In: InformationsbriefWeltwirtschaft \& Entwicklung 3-4: 2-3.

- (2015): Oligarch - eine Form bürgerlicher Herrschaft. Das Beispiel osteuropäischer semiperipherer Kapitalismus. In: PROKLA 45(3): 409-432.

Bojcun, Marko (2001): Russia, Ukraine and European Integration. EUI Working Paper HEC No. 4. URL: http://cadmus.eui.eu/bitstream/id/932/hec01-04.pdf/, Zugriff: 14.9.2015.

Böttger, Katrin (2014): Auf dem sicherheitspolitischen Auge blind: Die EU-Außenpolitik angesichts der Ukraine-Krise: Zustand und Entwicklungsoptionen. In: integration 2: 95-108.

Brand, Martin (2008): Die Europäische Nachbarschaftspolitik - ein neoliberales Projekt? In: UTOPIE kreativ H. 217: 997-106.

Brangsch, Lutz (2015): Die EU und ihre Nachbarn. In: Baier, Walter/Müller, Bernhard/Himmelstoss, Eva (Hrsg.): transform! Jahrbuch 2015 „Vereintes Europa, Geteiltes Europa“. Hamburg. URL: http://www.transform-network.net/de/zeitschrift/jahrbuch-2015/news/detail/Journal/ the-european-union-and-its-neighbours.html, Zugriff: 14.9.2015.

Council on Foreign Relations (1994): Budapest Memorandums on Security Assurances: URL: http:// www.cfr.org/nonproliferation-arms-control-and-disarmament/budapest-memorandumssecurity-assurances-1994/p32484, Zugriff: 14.9.2015.

Die Presse (2015a): Ausverkauf: Ukraine will 350 Staatsunternehmen privatisieren, 10.7.2015. URL: http://diepresse.com/home/wirtschaft/international/4774091/Ausverkauf_Ukrainewill-350-Staatsunternehmen-privatisieren?from=suche.intern.portal, Zugriff: 14.9.2015.

- (2015b): Ukraine: „Ich als Minister verdiene 200 Euro“, 09.10.2015. URL: http://diepresse. com/home/politik/aussenpolitik/4839169/Ukraine_Ich-als-Minister-verdiene-200-Euro, Zugriff: 14.10 .2015 .

EEAS (o.J.): EU financial and technical assistance for Ukraine: URL: http://www.eeas.europa.eu/ ukraine/about/financial-technical_assistance_en.htm, Zugriff: 14.9.2015.

EU (2015): Fact Sheet - Frequently asked questions about Ukraine, the EU's Eastern Partnership and the EU-Ukraine Association Agreement, URL http://www.eeas.europa.eu/statements/ docs/2014/140612_01_en.pdf, Zugriff: 14.9.2015.

EurActiv (2010): Russland/Ukraine: Neuer Deal mit Gas und Marinestützpunkt, 22.04.2010. URL: http://www.euractiv.de/erweiterung-und-nachbarn/artikel/russlandukraine-neuerdeal-mit-gas-und-marinesttzpunkt-002994, Zugriff: 14.9.2015.

EuroMemorandum (2015): Die Zukunft der Europäischen Union: Stagnation und Polarisierung oder eine grundlegende Neuausrichtung? URL: http://www2.euromemorandum.eu/uploads/ euromemorandum_2015_german.pdf

Euronews (2015): Ukraine crisis has created more than 2 million refugees, UN reports, 22.4.2015. URL: http://www.euronews.com/2015/04/22/ukraine-crisis-has-created-more-than-2-million-refugees-un-reports/, Zugriff: 14.9.2015.

Europäische Kommission (2009): Die Politik: Was ist die Europäische Nachbarschaftspolitik? URL: http://ec.europa.eu/world/enp/policy_de.htm, Zugriff: 14.9.2015.

- (2015): Ukraine as part of the European energy market - speech by Vice-President Šefčovič at the Energy panel at International Conference for Reform in Ukraine. URL: http://europa. eu/rapid/press-release_SPEECH-15-4888_en.htm, Zugriff: 14.9.2015.

GTAI (2015): Russland-Sanktionen aktuell, 23.6.2015. URL: http://www.gtai.de/GTAI/Navigation/DE/Trade/Maerkte/Specials/russland-sanktionen, $\mathrm{t}=1$-sanktionen-der-europaeischenunion-gegenueber-russland,did=1263744.html, Zugriff: 14.9.2015. 
Harvey, David (2003): The New Imperialism. Oxford.

Helmer, John (2015): The IMF is posturepedic, so Igor Kolomoisky can sleep well at night, 16.03.2015. URL: http://johnhelmer.net/?p=12944\&print=1, Zugriff: 14.9.2015.

Jopp, Mathias/Böttger, Katrin (2014): The Ukraine Crisis - Lessons for the EU. CIFE Policy Paper $n^{\circ}$.

Kubicek, Paul (2005): The European Union and democratization in Ukraine. In: Communist and Post-Communist Studies 38: 269-292.

Kyiv Post (2015): 'De-oligarchization' will recharge energy sector. In: Kyiv Post (Hrsg.): Doing Business in Ukraine: 26-18. URL: https://www.kyivpost.com/media/pdf/db.pdf, Zugriff: 14.9.2015.

Latifundist (2015): Ton 100 латифундистов Украиньь. URL: http://latifundist.com/rating/ top100\#96, Zugriff: 14.9.2015.

Mearsheimer, John J. (2014): Why the Ukraine Crisis Is the West's Fault. The Liberal Delusions That Provoked Putin. In: Foreign Affairs: 1-12.

Malek, Martin (2010): Die Beziehungen der EU zur Ukraine, zu Belarus, und zur Moldau. Sicherheitspolitische Aspekte vor dem Hintergrund des „russischen Faktors“. In. Strategie und Sicherheit 1: 167-182.

Matuszak, Slawomir (2012): The Oligarchic Democracy. The Influence of Business Groups on Ukrainian Politics (OSW Studies Nr. 42). Warsaw.

MFA (2012): Ukraine-EU Relations. URL: http://ukraine-eu.mfa.gov.ua/en/ukraine-eu/relations, Zugriff: 14.9.2015.

Müller, Klaus (2015): Warum die Ukraine gescheitert ist. Oligarchische Konsolidierung und geopolitisches Hasardspiel. In: PROKLA 45(1): 135-153.

Mykhnenko, Vlad/Swain, Adam (2010): Ukraine's diverging space-economy: The Orange Revolution, post-soviet development models and regional trajectories. In: European Urban and Regional Studies 17(2): 141-165.

Nationalbank der Ukraine (2015): Аані фінансовоїзвітності банків України, 01.7.2015. URL: http://www.bank.gov.ua/control/uk/publish/category?cat_id=64097, Zugriff: 14.9.2015.

NATO (2015): NATO's relations with Ukraine. URL: http://www.nato.int/cps/en/natohq/topics_37750.htm, Zugriff: 14.9.2015.

Pavlenko, Oleksiy (2015): Oleksiy Pavlenko: Reforms are happening in Ukraine's agrarian sector, 15.09.2015. URL: https://www.kyivpost.com/opinion/op-ed/oleksiy-pavlenko-reforms-arehappening-in-ukraines-agrarian-sector-397953.html, Zugriff: 14.9.2015.

Plank, Christina (2014a): Die politische Ökonomie der Oligarchen. In: Z.Zeitschrift Marxistische Erneuerung, Nr. 99: 44-52.

- (2014b): Die ‘inneren’ Dimensionen des Ukraine-Konflikt. In: Kurswechsel 3: 83-88.

- (2015): Liberalization of the Ukrainian Land Market: A Threat to the Right to Food, Right to Food and Nutrition Watch: 75-76. URL: http://www.rtfn-watch.org/fileadmin/media/ rtfn-watch.org/ENGLISH/pdf/Watch_2015/RtFNWatch_EN_web.pdf,ZZugriff: 14.9.2015.

-/Plank Leonhard (2014): The Financialisation of the Ukrainian Farmland. In: Journal für Entwicklungspolitik 30(2): 46-68.

Rachkevych, Mark (2015): Ukraine's agriculture minister breaks old habits of predecessors. In: Kyiv Post (Hrsg.): Doing Business in Ukraine: 14-16. URL: https://www.kyivpost.com/media/ pdf/db.pdf

Schubert, Samuel R./Pollak, Johannes/Brutschin, Elina (2014): Two futures: EU-Russian relations in the context of Ukraine. In: European Journal of Futures Research 2(52): 2-7.

SpiegelOnline (2012): Verkaufvon Tochterfirma: Commerzbank beendet Abenteuer in der Ukraine, 31.07.2012. URL: http://www.spiegel.de/wirtschaft/unternehmen/commerzbank-verkauftbank-forum-in-der-ukraine-a-847299.html, Zugriff: 14.9.2015. 
Spinner, Jackie (2005): Ukraine Announces Pullout of Iraq Force, The Washington Post, 11.01.2005. URL: http://www.washingtonpost.com/wp-dyn/articles/A64197-2005Jan 10.html, Zugriff: 14.9.2015.

Stern, David (2015): Ukraine conflict: Refugees in their own country, BBC News, 15.08.2015. URL: http://www.bbc.com/news/world-europe-33880351, Zugriff: 14.9.2015.

Szeptycki, Andrzej (2014): The European Union and Ukraine: Postcolonial Approach. In: International Relations 49(1): 181-201.

UN News Centre (2015): Close to 8,000 people killed in eastern Ukraine, says UN human rights report, 08.09.2015. URL: http://www.un.org/apps/news/story.asp?NewsID=51819\#.Ve_e5M5H9FU, Zugriff: 14.9.2015.

van der Pijl, Kees (2015): Kommt die globale autoritäre Oligarchie? Annäherungen und Konflikte zwischen dem Westen und dem Rest der Welt. In: Peripherie Nr. 137: 47-73.

Wolczuk, Kataryna (2009): Implementation without Coordination. The Impact of EU Conditionality on Ukraine under the European Neighbourhood Policy. In: Europe-Asia Studies, 61(2): 187-211.

Yurchenko, Yuliya (2012): „Black Holes“ in the Political Economy of Ukraine: The Neoliberalization of Europe's „Wild East“. In: Debatte: Journal of Contemporary Central and Eastern Europe 20(2-3): 125-149.

Zolinka, Marija (2015): Общественное мнение относительно НАТО: трендь, мотивации, предостережения, ZN.UA. URL: http://gazeta.zn.ua/international/obschestvennoe-mnenieotnositelno-nato-trendy-motivacii-predosterezheniya-_.html, Zugriff: 14.9.2015.

\section{VSA: Über Grenzen hinaus}

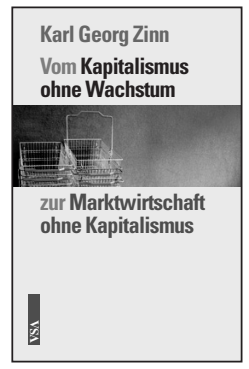

Karl Georg Zinn

Vom Kapitalismus

ohne Wachstum zur

Marktwirtschaft ohne Kapitalismus

168 Seiten I $€ 16.80$ ISBN 978-3-89965-

651-0

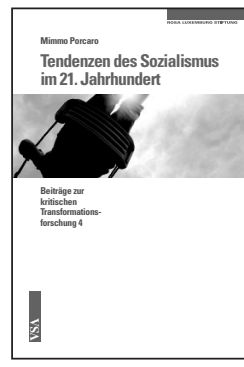

Mimmo Porcaro

Tendenzen des

Sozialismus im 21. Jahrhundert 120 Seiten $\mid € 9.80$ ISBN 978-3-89965-

676-3

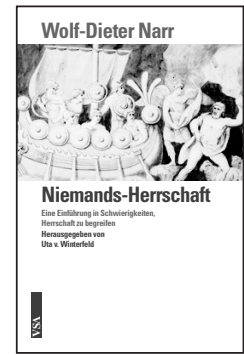

Wolf-Dieter Narr Niemands-Herrschaft Eine Einführung in Schwierigkeiten, Herrschaft zu begreifen 320 Seiten I $€ 26.80$ ISBN 978-3-89965600-8
Der Sozialismus kommt

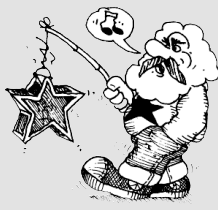

kritisch links

Analysen Positionen Berichte monatlich gedruckt Jahresabo $€$ 70,(€ 50,- ermäßigt) täglich im Netz www. sozialismus.de

\section{www.vsa-verlag.de}

